\title{
PERSPECTIVE
}

\section{Birdshot retinochoroidopathy}

\author{
Alice T Gasch, Janine A Smith, Scott M Whitcup
}

Birdshot retinochoroidopathy is a rare, chronic, bilateral, posterior uveitis with a distinctive clinical presentation and a strong genetic association. Middle aged white people of northern European extraction are most commonly afflicted. ${ }^{1-4}$ The pathogenesis is unknown, but HLA-A29 positivity appears to confer predisposition, ${ }^{5-9}$ and retinal autoimmunity seems to play a role. ${ }^{8}$

This review provides an updated summary of the current state of knowledge about birdshot retinochoroidopathy. It includes results of visual field and electrophysiological testing from markedly more patients than previous reports of these data. Furthermore, it provides prognostic information regarding visual acuity based on nearly twice the number of patients than the one previous report of this aspect of the disease. Such information is of special interest to patients. The paper is based on a chart review of the 59 birdshot patients evaluated at the National Eye Institute (NEI) and from a comprehensive review of the literature.

\section{Historical background}

Perhaps the earliest report of birdshot retinochoroidopathy was in 1949 by Franceschetti and Babel, who named it descriptively "la chorio-rétinite en 'täches de bougie" candle wax spot chorioretinopathy. ${ }^{10}$ Subsequently, other descriptive terms have been used-salmon patch choroidopathy, ${ }^{11}$ "choriorétinopathie en grains de riz" (that is, rice grain chorioretinopathy), ${ }^{12}$ vitiliginous chorioretinitis, ${ }^{1}$ and birdshot retinochoroidopathy. ${ }^{4}$ The term "vitiliginous chorioretinitis" was chosen both because the hypopigmented fundus lesions resemble vitiligo and because some individuals in one cohort of birdshot patients had cutaneous vitiligo. However, this systemic association has been noted by only one researcher. ${ }^{1}$ The term "birdshot retinochoroidopathy" is most commonly used. It was coined by Ryan and Maumenee in 1980 to describe the fundus lesions, which typically consist of "multiple, small, white spots that frequently have the pattern seen with birdshot in the scatter from a shotgun." The disease became accepted as a distinct entity in the early 1980s.

\section{Epidemiology}

Birdshot retinochoroidopathy is rare. In the United States, at one uveitis clinic, seven of 600 patients (1.2\%) were diagnosed with the disease..$^{13}$ In Europe, at 14 eye clinics, 102 cases were diagnosed collectively between 1980 and $1986 .^{3}$ Fifty nine cases have presented to the NEI since and including 1980 .

Birdshot occurs at a later age than many types of uveitis. Average patient age according to the literature is about 50 years with a range of 35-70 years. ${ }^{1-4}$ At the time of diagnosis the mean age of 56 of the NEI patients was 48.2 (SD 9.9) years (range $24-71$ years). Age at diagnosis was not available for three patients.

White people of northern European descent are the most frequently afflicted racial and ethnic group. ${ }^{1-4}$ Fifty
Table 1 Patient characteristics of the NEI birdshot population ( $n=59)$

\begin{tabular}{ll}
\hline Age at diagnosis (years) & \\
Mean (SD) & $48.2(9.9)$ \\
Range & $24-71$ \\
Race: & \\
White & 58 \\
Hispanic & 1 \\
Sex: & \\
Male & 25 \\
Female & 34 \\
\hline
\end{tabular}

${ }^{\star}$ Data unavailable for three patients.

eight of our 59 patients were white and one was Hispanic. A sex predilection for women has been reported in some, ${ }^{14}$ but not all, ${ }^{74-16}$ studies; $58 \%$ of the NEI population were women (Table 1).

Although association of birdshot with other ocular and systemic diseases has been reported, typically patients are otherwise relatively healthy, and the reported associations may be spurious findings. An unusually high incidence of glaucoma was found in one study $(19 \%, 15$ eyes $),{ }^{3}$ but has not been corroborated. Rhegmatogenous retinal detachment also has been reported in association with birdshot, ${ }^{241718}$ but there is evidence that the incidence is not increased. ${ }^{3}$ In addition, vascular disease, ${ }^{3}$ sarcoidosis, ${ }^{3}{ }^{19}$ psoriasis, ${ }^{20}$ autoimmune sensorineural hearing loss, ${ }^{21}$ and, as noted above, vitiligo ${ }^{1}$ have been reported in birdshot patients.

Table 2 shows all other ocular conditions reported by the NEI population, and Table 3 shows non-ocular problems reported by more than one patient in the NEI population. Of the former, ocular hypertension was most common, and in most cases it was presumed secondary to steroid use. Systemic hypertension was the most commonly reported non-ocular problem. Twenty per cent of the NEI population reported idiopathic systemic hypertension. This prevalence is well below that found in the Framingham study in which a similar population has been studied (that is, white suburbanites). In the Framingham study, almost half of the subjects have had blood pressure repeatedly over $140 / 90 \mathrm{~mm} \mathrm{Hg} .{ }^{22}$ Although the list of non-ocular problems in NEI birdshot patients is long, a minority of the patients had multiple problems. Thirty four per cent had no history of a significant disease and $75 \%$ had no significant systemic disease concomitant with birdshot.

Table 2 Ocular conditions other than birdshot retinochoroidopathy in the NEI birdshot population

Ocular hypertension

Amblyopia

High myopia

Retinal tear/hole (1 patient had high myopia)

Dry eye

Blephariti

Meibomianitis

Central serous retinopathy

Non-ischaemic branch retinal vein occlusion

Band keratopathy

Ocular migraine

${ }^{\star}$ Number of patients with the condition. 
Table 3 Non-ocular diseases reported by more than one patient in the NEI birdshot population

Systemic hypertension (12 idiopathic, 3 secondary to cyclosporine) $15^{\star}$ Arthritis (4 unspecified, 2 osteoarthritis)

Hypercholesterolaemia

Cardiac arrhythmia

Diabetes mellitus

Hypacusis (attributed to loud noise)

Migraine headache

Hiatal hernia

Coronary artery disease ( 1 status post coronary artery bypass grafting, 1 status post myocardial infarction)

Mitral valve prolapse

Gout

Diverticulitis

Colitis

Irritable bowel syndrom

Scoliosis

${ }^{\star}$ Number of patients with the condition.

Table 4 Ocular symptoms of the NEI birdshot population

"Decreased," "blurry," "hazy," "foggy," or "grey" vision $\quad 40^{\star}(68 \%)$

Floaters

Nyctalopia

Dyschromatopsia

Glare

Photopsia

Light sensitivity

"Black spots"

"Blind spot"

Fluctuating vision

Pain

Decreased depth perception

"Shimmering" vision

Metamorphopsia

Decreased peripheral vision

Light adaptation difficulty

$\star$ Number of patients with the symptom.

\section{Clinical features}

TYPICAL PRESENTATION

Some NEI patients were asymptomatic at the time of diagnosis and some were symptomatic before signs of the disease appeared. The most common complaints of the NEI population were decreased vision $(68 \%)$, floaters $(29 \%)$, nyctalopia (25\%), dyschromatopsia (20\%), glare (19\%), and photopsia $(17 \%)$. Other less frequent symptoms are listed in Table 4. It has been reported that visual complaints are often out of proportion to measured visual acuity. ${ }^{11}$

Diagnostic criteria formulated by Ryan and Maumenee, who first described a cohort of patients, are: (1) minimal, if any, anterior segment inflammation; (2) diffuse vitritis without snowbanking; (3) retinal vascular leakage, particularly in the posterior pole, which may be associated with secondary cystoid macular oedema and optic disc oedema; (4) distinctive, discrete, cream coloured or depigmented spots throughout the postequatorial fundus; and (5) a painless eye. ${ }^{4}$ Subsequently, Priem and Oosterhuis, who reported the largest series of 102 cases, used the diagnostic criteria of: (1) bilateral birdshot spots plus (2) retinal vasculopathy or disc oedema or optic atrophy or vitreous cells. $^{3}$

The NEI population, which comprised some patients with active disease and others with inactive disease, all exhibited: (1) minimal, if any, anterior segment inflammation; (2) vitritis without snowbanking and/or vitreous debris; and (3) typical birdshot lesions (described below) bilaterally or typical birdshot lesions unilaterally in the setting of a middle aged white person of northern European extraction with HLA-A29 positivity and both blue-yellow dyschromatopsia and nyctalopia in the eye without lesions. One NEI patient had unilateral lesions, which will be discussed below.

Typically, few signs of anterior segment inflammation are present. It has been stated that posterior synechiae are never observed. ${ }^{11}$ However, three of the NEI patients had posterior synechiae (one unilateral, two bilateral).

Vitritis varies in severity among patients and over time. Often it is most severe early in the disease. ${ }^{11}$

The typical fundus lesions are subretinal, poorly defined, cream coloured spots without reactive hyperpigmentation, about one quarter to three quarters of an optic disc diameter in size. ${ }^{11}$ Over time lesions may become confluent and/or hyperpigmented. Eventually, lesions may become well delineated, white atrophic spots, involving the retina.

The lesions are distributed in the posterior pole and mid-periphery, and the following four patterns of distribution have been described and named descriptively: (1) diffuse, (2) macular sparing, (3) macular predominance, and (4) asymmetric. In the "asymmetric" pattern there is concentration of the spots in the inferonasal fundus with relative macular sparing. ${ }^{18}$

Bilaterality of lesions is usual, though involvement may be asymmetric. However, initially only one eye may exhibit lesions. Thus, we made provision for diagnosing the disease in NEI patients with unilateral lesions.

Occasionally, the fundus spots appear several years after onset of concomitant vitritis, retinal vasculitis, and papillitis. $^{323}$ Consequently, the disease may be misdiagnosed as idiopathic retinal vasculitis. ${ }^{23}$

Arteriolar narrowing is common, and vascular tortuosity is not uncommon. ${ }^{4}$

SEQUELAE

Table 5 shows the frequency of sequelae in the largest reported series of 102 cases $^{3}$ and in the NEI population. Cystoid macular oedema was by far the most frequent complication in both series. Optic disc oedema and epiretinal membranes were the next most common complications. Macular pucker secondary to the latter may progress despite resolution of inflammation with therapy. ${ }^{16}$

Table 5 Sequelae of birdshot retinochoroidopathy

\begin{tabular}{|c|c|c|}
\hline Sequela & Priem and Oosterhuis ${ }^{3}$ (No of eyes $=54$ ) & NEI population (No of eyes $=116$ ) \\
\hline $\begin{array}{l}\text { Cystoid macular oedema } \\
\text { Non-cystoid macular oedema }\end{array}$ & $\begin{array}{l}33(61 \%) \\
\dagger \\
\left.\text { Priem and Oosterhuis }{ }^{3} \text { (No of eyes }=203\right)\end{array}$ & $\begin{array}{l}36(31 \% \text { eyes; } 37 \% \text { patients }) \\
6(7 \% \text { patients })\end{array}$ \\
\hline Optic disc oedema & $t$ & 14 ( $12 \%$ eyes; $14 \%$ patients $)$ \\
\hline Optic nerve atrophy & 7 & 2 (1 patient) \\
\hline \multicolumn{3}{|l|}{ Subretinal neovascular membrane } \\
\hline Peripapillary & 4 & 0 \\
\hline Macular & 8 & 4 (3 patients) \\
\hline Macular scar & $\dagger$ & 2 (2 patients) \\
\hline Subretinal fibrosis (peripapillary) & $t$ & 2 (1 patient) \\
\hline Retinal neovascularisation & 15 & 3 (2 patients) \\
\hline Vitreous haemorrhage & 5 & 1 \\
\hline Epiretinal membrane & 20 & 11 (7 patients) \\
\hline
\end{tabular}

* Two NEI patients underwent unilateral enucleation for complications following cataract surgery. 
Table 6 Laser flare photometry results in birdshot retinochoroidopathy

\begin{tabular}{lrl}
\hline Disease activity & Birdshot patients & Controls $(n=88)$ \\
\hline Not stated $(\mathrm{n}=4)$ & $5.7(1.1)^{\star} \dagger$ & $4.7(0.16) \dagger$ \\
Active $(\mathrm{n}=7)$ & $9.6(2.0) \ddagger$ & \\
Inactive $(\mathrm{n}=3)$ & $11.6(2.8) \ddagger$ & \\
\hline
\end{tabular}

${ }^{\star}$ Mean (SE).

†Guex-Crosier et al. ${ }^{28}$

$\ddagger$ NEI population.

Table 7 Visual field testing abnormalities in the NEI birdshot population

\begin{tabular}{ll}
\hline & No of eyes tested (32) \\
\hline Enlarged blind spot & 15 \\
Paracentral and/or midperipheral islands of scotoma & 9 \\
Generalised decreased sensitivity & 4 \\
Constriction & 0 \\
\hline
\end{tabular}

Subretinal neovascular membranes occur infrequently. They are important to identify because they can cause permanent vision loss as a result of haemorrhage and scar formation that can be prevented in some cases by prompt treatment (usually with laser photocoagulation). As well as involving juxtafoveal and juxtapapillary areas, ${ }^{3414}$ they can develop adjacent to fundus lesions 6 months to 5 years after the onset of birdshot. ${ }^{13}$

Retinal neovascularisation also occurs infrequently. Secondary vitreous haemorrhage may reduce vision. Retinal neovascularisation may involve the peripapillary area or periphery. ${ }^{325}$ It may be induced by local inflammation in the retinal vascular bed. ${ }^{26}$ Because it occurs in the absence of retinal capillary non-perfusion, it does not appear to be a response to ischaemia. ${ }^{3}$

Optic nerve atrophy occurs in a small minority of patients. Typically, it is an end stage sequela, presumed secondary to long standing retinal inflammation. ${ }^{24}$ There has been one report of it occurring secondary to acute anterior ischaemic optic neuropathy. ${ }^{27}$
LASER FLARE PHOTOMETRY

Laser flare photometry results (Table 6) were not significantly different between four birdshot patients (with unspecified degree of disease activity) and 88 controls $^{28}$ or between six eyes of three inactive patients and 14 eyes of seven active patients. These findings are consistent with the minimal signs of inflammation in the anterior chamber seen clinically.

VISUAL FIELD TESTING

Reports of visual field testing are limited. Amsler grid testing revealed metamorphopsia in one or both eyes of all of five patients tested. ${ }^{1}$ Goldmann perimetry performed on five patients was normal in one patient (with diffuse birdshot fundus lesions), and showed peripheral field constriction in four patients, two of whom also had enlarged blind spots. One of these two patients had peripapillary scotomas corresponding to depigmented lesions. Otherwise, birdshot fundus lesions were not associated with field abnormalities. ${ }^{1}$ Visual field testing by confrontation was normal in two patients tested. ${ }^{1}$

In another report, visual fields were full in four of six patients tested, depressed peripherally in one patient, and constricted peripherally due to a retinal detachment in another patient. The means of testing was not specified. ${ }^{18}$

Amsler grid testing data were available for eight NEI patients. It was normal for one, revealed a scotoma in two (bilateral in one, unilateral and associated with metamorphopsia in the other), and revealed metamorphopsia without a scotoma in five (bilateral in one).

Humphrey or Goldmann visual field testing data was available for 16 other NEI patients. (Table 7). In contrast with previous reports, ${ }^{8} 18$ peripheral visual field constriction was not evident. The most common finding was an enlarged blind spot associated either with optic disc oedema or a circumpapillary birdshot lesion. Another common finding was small islands of paracentral and/or
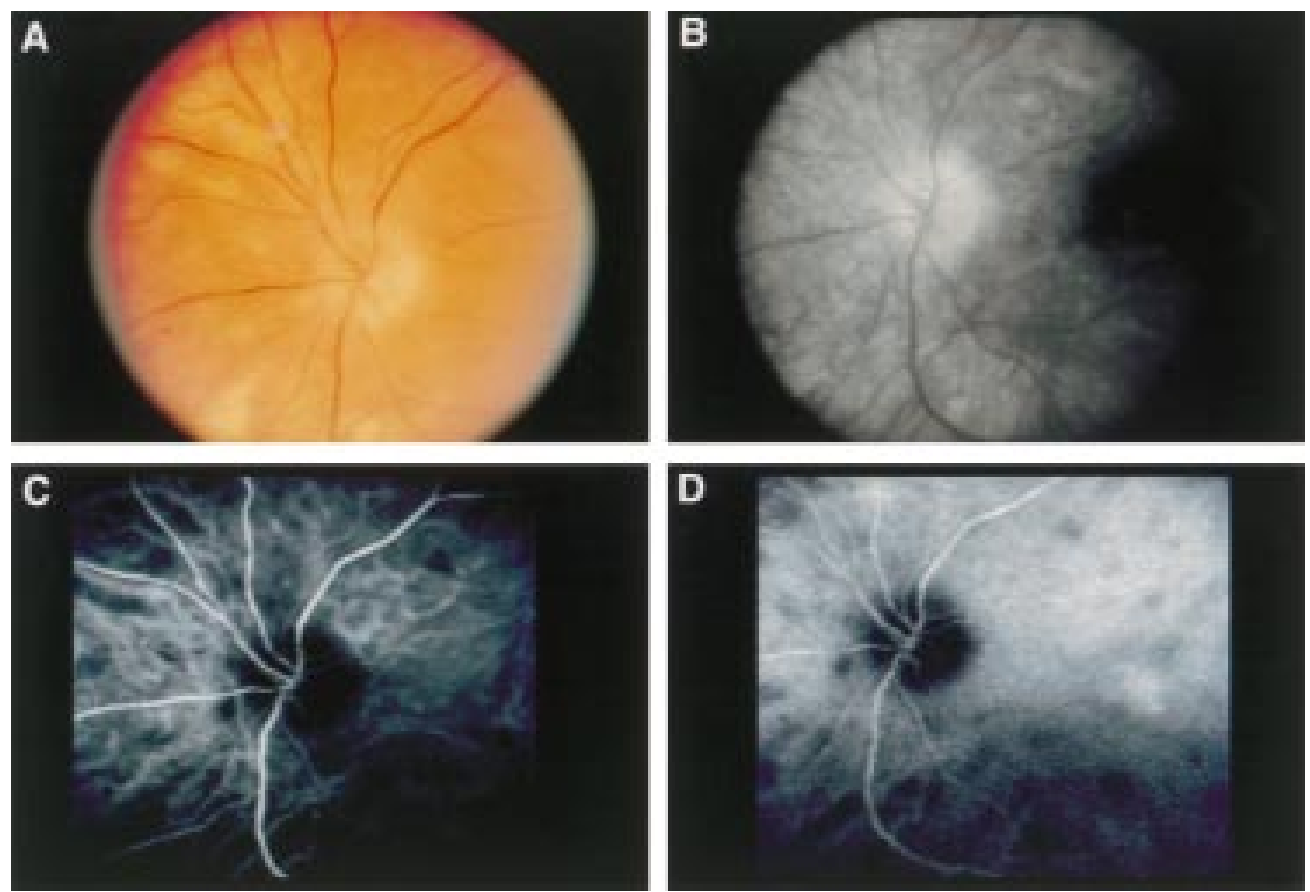

Figure 1 (A) Photograph of the left peripapillary fundus of a patient with short standing birdshot retinochoroidopathy, showing relatively few birdshot lesions and optic disc oedema. (B) Late phase of fluorescein angiography of the same eye, demonstrating silence of birdshot lesions. Optic disc leakage also is evident. (C) and (D) Early and late phases respectively of indocyanine green angiography of the same eye, revealing more birdshot lesions than ophthalmoscopy or fluorescein angiography. The lesions manifest early in the study and persist essentially unchanged. There is no sign of the optic disc oedema evident with fluorescein angiography. 

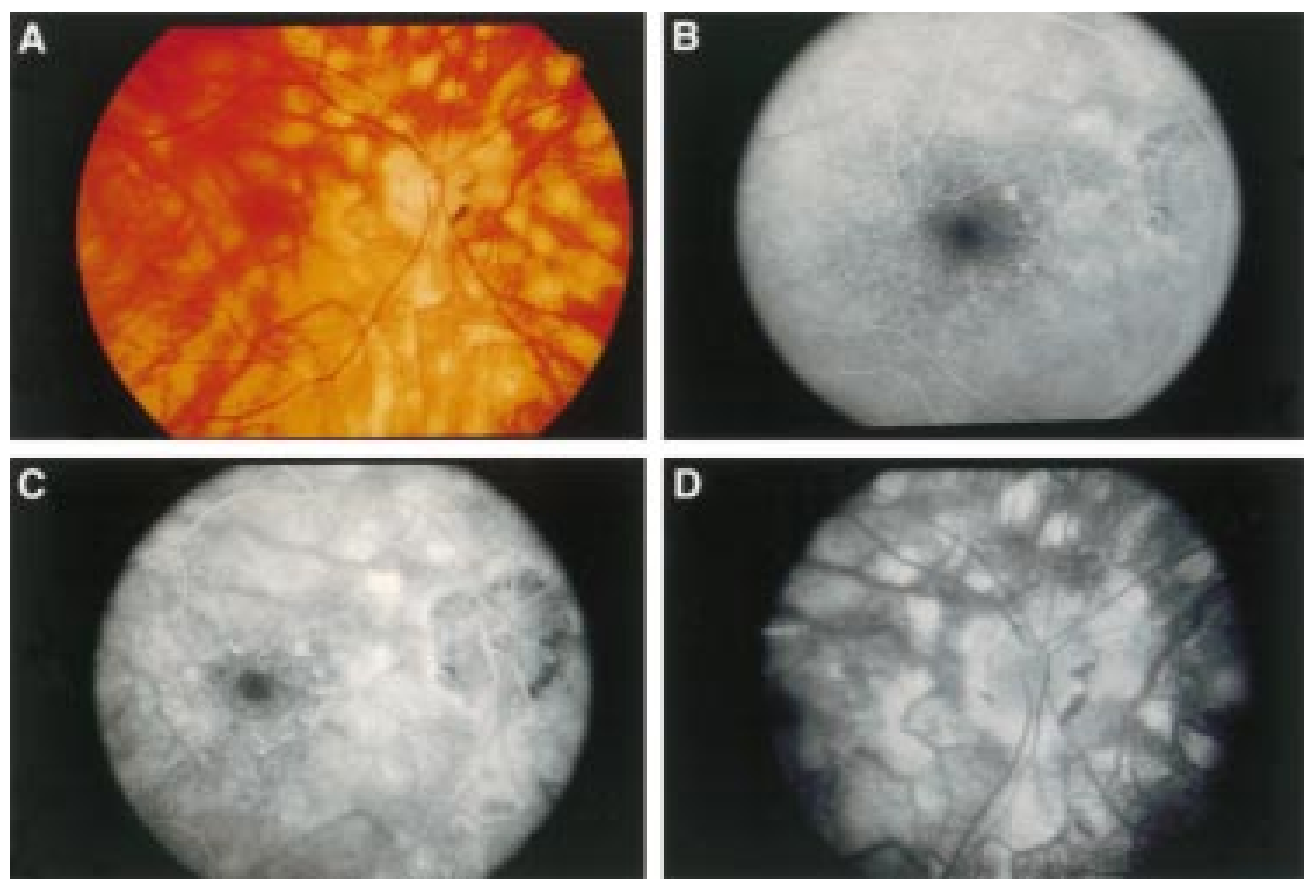

Figure 2 (A) Photograph of the right posterior pole of a patient with long standing birdshot retinochoroidopathy, showing diffuse birdshot lesions and circumpapillary retinal atrophy. (B), (C), and (D) Early, mid, and late phases respectively of

fluorescein angiography of the same eye, demonstrating staining of birdshot lesions.

mid-peripheral scotomas, which did not necessarily correspond with birdshot lesions. Generalised diminished sensitivity alone also was found. Visual fields were normal in two patients, both of whom had diffuse birdshot lesions and underwent Goldmann visual field tests. Thus, visual field testing is not necessarily abnormal, even if there are diffuse birdshot lesions.

\section{INDOCYANINE GREEN ANGIOGRAPHY}

Indocyanine green (ICG) angiography is not a useful management tool for birdshot retinochoroidopathy because it is a poor indicator of disease activity and sequelae. ${ }^{29}$ However, it is useful diagnostically because of the unique character of birdshot lesions on ICG angiography.

ICG angiography reveals more lesions than either ophthalmoscopy or fluorescein angiography (FA). Lesions appear as well delineated, hypofluorescent spots, similar in size to clinical lesions, and less numerous in the macula than the mid-periphery. The spots manifest early (within 5 minutes of dye injection) and persist unchanged throughout the study ${ }^{29}$ (Fig 1). Often the spots are distributed along choroidal vessels, suggesting a choroidal vasculitis. ${ }^{29}$

Reportedly, lesions predominate at the mid-choroidal level, where there is a cleavage plane between the middle and outer choroid (that is, Sattler's and Haller's layers). It has been proposed that inflammatory exudate infiltrates the cleavage plane and undergoes fibrosis, thereby fusing the choroidal interstitium and resulting in atrophy. ICG molecules may not conjugate with the inflammatory infiltrate, resulting in hypofluorescent spots. Focal choroidal atrophy could explain the persistence and expansion of the hypofluorescent spots over time. ${ }^{29}$

\section{FLUORESCEIN ANGIOGRAPHY}

Unlike ICG angiography, FA is a useful management tool for birdshot retinochoroidopathy because it facilitates assessment of sequelae which may require treatment, including cystoid macular oedema and subretinal neovascular membranes. ${ }^{29}$

On FA, fundus lesions exhibit heterogeneity. It has been suggested that this phenomenon may be explained at least partly by lesion progression as follows. Initially, lesions may remain silent throughout the angiogram if they do not affect the retinal pigment epithelium (RPE). Thus, more spots may be appreciated clinically than on FA (Fig 1B). Later, there may be staining within and then throughout spots seen clinically as the RPE becomes increasingly affected (Fig 2). Lesions of different stages may coexist in the same fundus, resulting in angiographic heterogeneity within one eye. ${ }^{11}$

\section{ELECTROPHYSIOLOGICAL TESTING}

Electrophysiology findings indicate varying degrees of middle retina, photoreceptor, and possibly RPE involvement.

The following has been proposed regarding the electroretinogram (ERG). When retinal involvement is mild, the ERG may be supernormal, possibly as a result of retinal irritability secondary to inflammation. ${ }^{30}$ With disease progression, the ERG b-wave amplitude and then the a-wave amplitude progressively diminish until they become non-recordable. ${ }^{30}$ The b-wave is generated by the middle/ neural network of the retina (that is, Mueller and bipolar cells), and the a wave is generated by photoreceptors. Thus, there may be initial compromise of Mueller and bipolar cells, followed by photoreceptor compromise. It has been noted that selective b-wave reduction with awave preservation, which suggests impairment of the neural network of the retina with little or no involvement of the photoreceptor-RPE-choroid complex, ${ }^{30}{ }^{31}$ is not typical of other uveitides. $^{30}$

In all 22 NEI patients tested, the ERG was abnormal bilaterally, though not necessarily symmetrically. In all cases either the b-wave amplitude or both the b- and a-wave amplitudes were reduced. In no case was the a-wave amplitude alone diminished (Fig 3). In general, rod mediated responses were equally or more compromised than those of cones (Fig 4).

Furthermore, most birdshot patients have a subnormal electro-oculogram (EOG), which could be due to photoreceptor and/or RPE dysfunction (Fig 5). The Arden ratio (light peak amplitude $\div$ dark trough amplitude) derived 

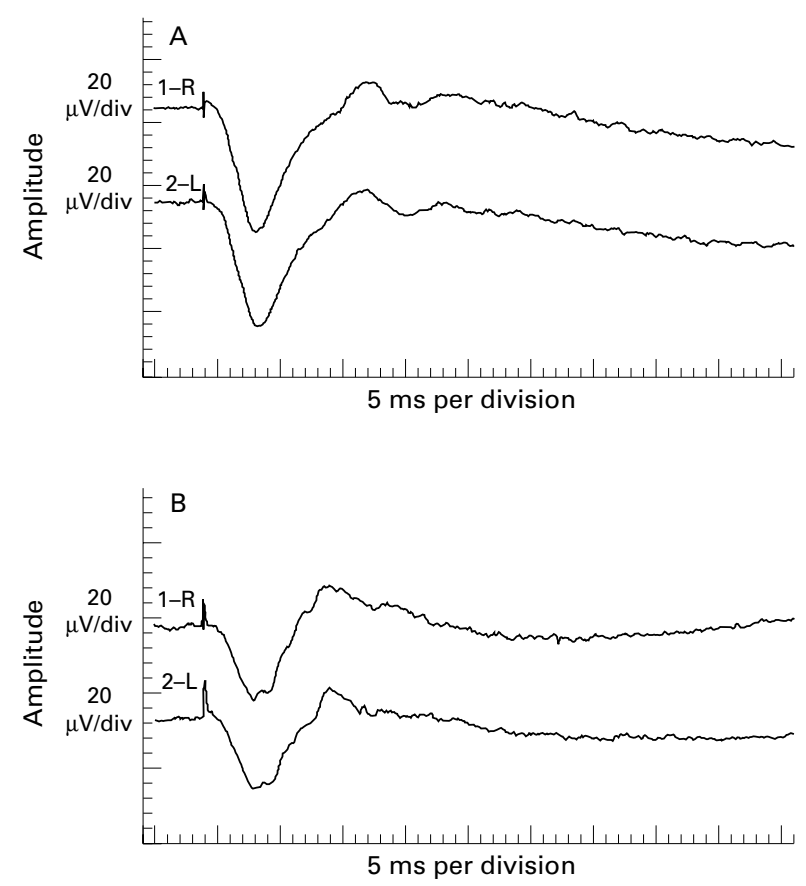

Figure 3 Electroretinogram maximal retinal responses of two patients with birdshot retinochoroidopathy. (A) A normal a-wave amplitude and moderately reduced b-wave amplitude, resulting in an abnormally low $b$ to a-wave ratio, suggests more compromise of intermediate than outer retinal layers. (B) Equally reduced $a$ - and b-wave amplitudes, resulting in a normal b- to a-wave ratio, suggests photoreceptor involvement.

from the EOG was subnormal in about $50 \%$ to $76 \%$ of patients in the literature. ${ }^{1230}$ In the NEI population 10 of the 11 patients who underwent electro-oculography had a subnormal EOG bilaterally. Eight patients who underwent an EOG had a concomitant ERG, and it was abnormal in all cases. The one patient with a normal EOG had an abnormal ERG with a normal a-wave amplitude, suggesting neural retina compromise with little, if any, photoreceptor or RPE involvement. This patient had been diagnosed with birdshot 1 month before electrophysiological testing, and visual acuity was $20 / 20$ bilaterally.

Dark adaptation threshold was elevated in about $71 \%$ to $80 \%$ of patients in the literature, ${ }^{3} 30$ indicating compromised rods and/or retina neural network, which is consistent with ERG findings. In the NEI population dark adaptation threshold was elevated in all 13 patients tested (bilaterally in eight). Two patients underwent subsequent retesting 5 years later and exhibited further elevation of the dark adaptation threshold.

Pattern visual evoked cortical potential (VEP) abnormalities are common, ${ }^{3}$ but probably are due to factors other than optic nerve dysfunction (for example, macular oedema $)^{31}$ in most birdshot patients. This factor was evaluated in only one NEI birdshot patient, whose ERG indicated bilateral focal or multifocal rod and cone dysfunction. The VEP exhibited prolonged latency to all stimuli, but these abnormalities were attributable to the patient's retinal disease because of her ERG findings.

\section{COLOUR VISION TESTING}

The predominant colour defect in both Priem and Oosterhuis' series ${ }^{3}$ and the NEI patients tested was blue-yellow (Table 8), which is consistent with acquired dyschromatopsia.

\section{Histopathological features}

Pathological evaluation of eyes with birdshot retinochoroidopathy has been limited to one eye of a 49 year old patient. The eye became phthisical after extraction of the
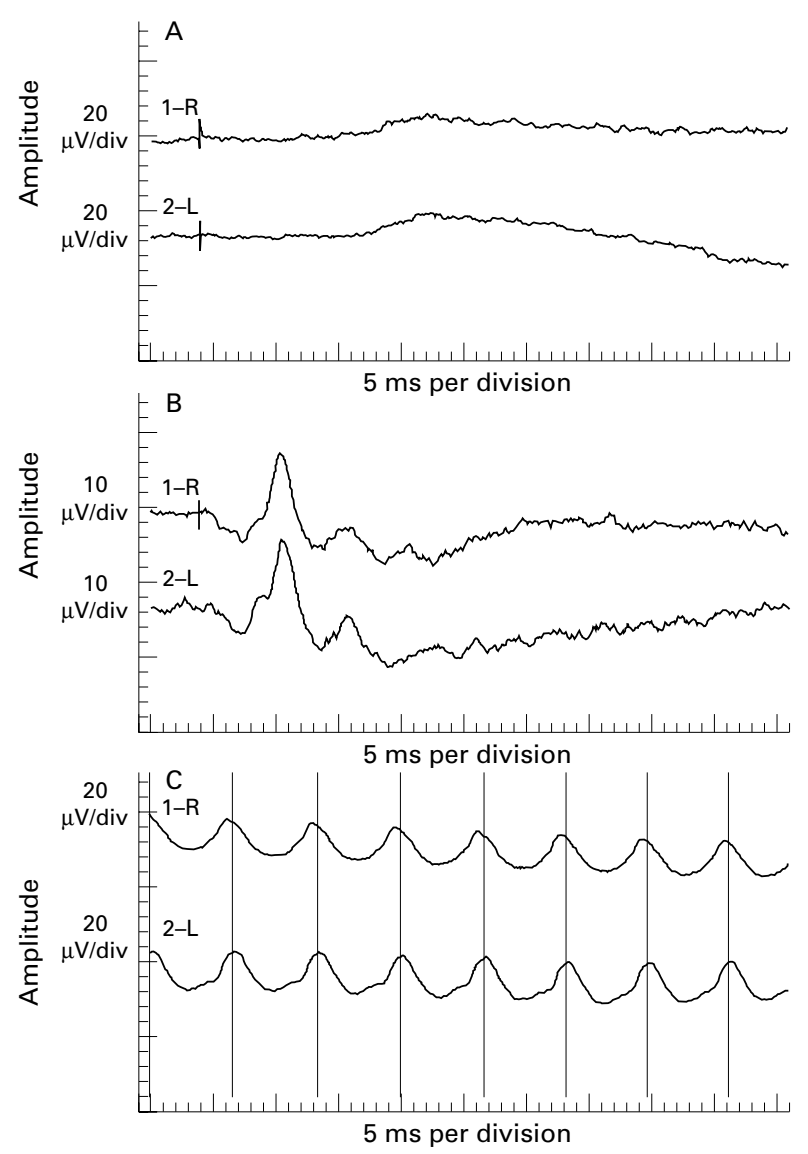

Figure 4 Electroretinogram rod mediated, cone mediated, and ficker. responses of the patient whose maximal retinal response is shown in Figure 3B. (A) Rod mediated responses have a reduced amplitude. (B) Cone mediated responses have a less reduced amplitude. (C) Flicker responses, which cones generate, have a reduction in amplitude equal to that of the cone mediated responses.

lens, which had subluxed into the anterior chamber with resultant elevation of intraocular pressure. The retina and choroid exhibited diffuse, chronic, granulomatous inflammation with epithelioid cells, giant cells, lymphocytes, and plasma cells. The retina was diffusely atrophic, whereas the choroid was not-possibly due to more intense inflammation in the retina than the choroid. Thus, it was suggested that the inflammation of the choroid might have been secondary to that of the retina. ${ }^{8}$

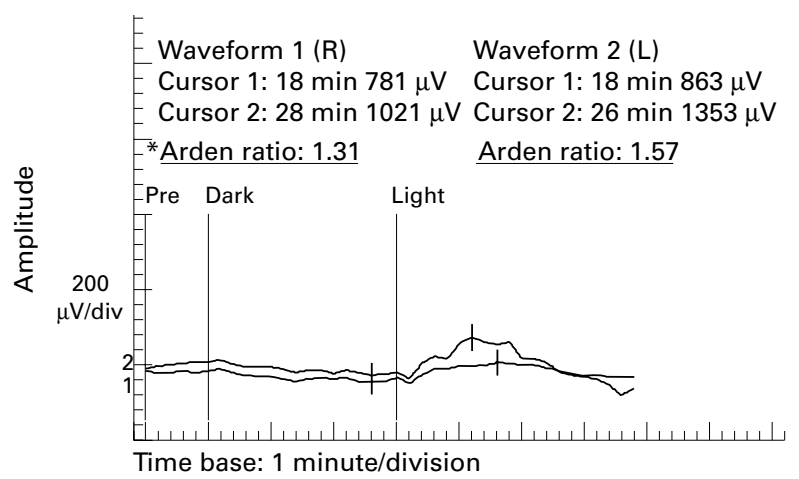

Figure 5 Electro-oculogram of a patient with birdshot retinochoroidopathy. Dark trough and light peak amplitudes and light peak implicit times are within the normal range for both eyes. The Arden ratio is below the normal range for the right eye and at the low end of the normal range for the left eye. ${ }^{\star N}$ Normal Arden ratio: mean 2.51 (SD 0.48 ); range 1.56-3.46. 
Table 8 Colour vision defects in birdshot retinochoroidopathy

\begin{tabular}{lll}
\hline Colour vision defect & $\begin{array}{l}\text { Priem and Oosterhuis } \\
\text { (56 eyes) }\end{array}$ & $\begin{array}{l}\text { NEI population* } \\
\text { (35 eyes) }\end{array}$ \\
\hline Blue-yellow & $41 \%$ & $57 \%$ \\
Blue-yellow and red-green & $21 \%$ & $29 \%$ \\
Red-green & Not stated & $9 \%$ \\
Normal & $38 \%$ & $6 \%$ \\
\hline
\end{tabular}

^ Total is $101 \%$ owing to numerical rounding to nearest full unit.

\section{Pathogenesis}

The pathogenesis of birdshot retinochoroidopathy is unclear. There is no identifiable mode of inheritance. HLA-A29 positivity appears to confer predisposition, and retinal autoimmunity seems to play a prominent role.

There is a strong association between birdshot retinochoroidopathy and HLA-A29 antigen. This antigen is present in about $80-98 \%$ of birdshot patients versus about $7 \%$ of controls, and increases risk of the disease about 50-224 fold..$^{5-9}$ It is composed of two subtypes: A29.1 and A29.2. The distribution of the subtypes varies between ethnic groups. Among HLA-A29 positive white people, about $80-90 \%$ have the HLA-A29.2 subtype. ${ }^{152}{ }^{32}$ It is this subtype that predominates in birdshot patients. ${ }^{7}{ }^{15}$

HLA-A29.2 may predominate in birdshot patients because it is more common in the population in which birdshot occurs (that is, white people) ${ }^{15}$ or because it imparts susceptibility to the disease. ${ }^{7}$ HLA-A29.1 and A29.2 differ by a single factor in the extracellular domain. ${ }^{32}$ It has been proposed that A29.1 is a mutation of A29.2 and that the mutation confers resistance to birdshot by inhibiting interaction with cytotoxic $\mathrm{T}$ lymphocytes and concomitantly promoting interaction with an accessory molecule (for example, CD8), thereby inhibiting $\mathrm{T}$ lymphocyte activation. ${ }^{33}$ However, any resistance conferred is not complete because one patient in the literature $^{15}$ and at least one NEI patient were HLA-A29.1 positive.

In the NEI population 39 patients were HLA-A29 positive, one for A29.1 and 18 for A29.2. Information regarding subtype was unavailable for the other 20 HLA-A29 positive patients. No patient was known to be HLA-A29 negative; however, HLA data were not available for 20 patients.

Although birdshot has been reported in a pair of HLAA29 positive twins, ${ }^{34}$ there is no identifiable mode of inheritance for the disease. ${ }^{11}$

Involvement of an infectious agent has been proposed because there are clusters of birdshot patients in specific areas of Holland, Belgium, and France. An infection could facilitate presentation of self peptides to $T$ cells by the HLA-A29 molecule with resultant autoimmunity ${ }^{1535}$ as follows: HLA class I proteins (including HLA-A29) may play a role in destroying virus infected cells. Viral peptides may bind between two alpha helices of the HLA-A29.2 molecule. When such a hybrid molecule reaches the cell surface, it could contact cytotoxic $\mathrm{T}$ lymphocytes having a receptor that fits both an epitope of the viral peptide and the alpha helices of the HLA-A29.2 molecule, the result being $T$ cell activation resulting in lysis of the peptide presenting cell and possibly initiation of autoimmune disease. ${ }^{24}$

There are at least three sources of evidence for retinal autoimmunity. (1) The lymphocytes of many patients exhibit an in vitro, cell mediated response to retinal $S$ antigen, ${ }^{78}$ particularly immediately before relapse of ocular inflammation. ${ }^{36}$ In the NEI population 11 patients exhibited this response, nine did not, and data were unavailable for 38. (2) The inflammatory response observed histologically (see "Histopathological features" above) appeared to be directed primarily to the retina, ${ }^{8}$ where photoreceptors and retinal $\mathrm{S}$ antigens are located. (3) There are numerous similarities between birdshot and retinal $S$ antigen induced autoimmune uveitis (EAU) in animals. ${ }^{37-40}$ These observations, combined with the strong HLA association, support an inbred potential for immune dysregulation involving the retina.

However, the trigger and pathogenic role of retinal autoimmunity are unclear. Retinal autoimmunity may be an epiphenomenon that develops after retinal damage has occurred or it may perpetuate inflammation that has already been initiated. ${ }^{26}$

Furthermore, there may be a systemic component to the disease. Systemic involvement is suggested by the discovery of higher serum levels of soluble IL-2 receptor in active birdshot patients than in controls. ${ }^{11}$ In addition, the pineal gland and the retina have common antigens because they have a common embryological origin. ${ }^{41}$ Because the pineal gland secretes melatonin, which controls dermal pigmentation, pineal gland autoimmunity concomitant with birdshot could explain the reported cases of birdshot associated with cutaneous vitiligo. ${ }^{42}$

\section{Diagnosis}

The diagnosis of birdshot retinochoroidopathy is made clinically. ${ }^{4}$ The prototypical patient with active disease is an otherwise healthy, middle aged white person of northern European descent, who has bilateral chronic decreased vision, floaters, nyctalopia, dyschromatopsia, relatively quiet anterior segments, vitritis without snowbanking, characteristic birdshot fundus lesions (that is, deep, poorly defined, cream coloured lesions about one quarter to three quarters of a disc diameter in size in the posterior pole), and possibly cystoid macular oedema and/or optic disc oedema and/or retinal vasculitis and/or arteriolar attenuation ( see "Clinical features" above).

Tests can help make the diagnosis in unclear cases. HLA-A29 typing has an estimated sensitivity of $96 \%$ and specificity of $93 \% .{ }^{43}$ Typically, other laboratory studies are normal, but can be valuable in ruling out other conditions in the differential diagnosis.

ICG angiography, fluorescein angiography, electroretinography, electro-oculography, dark adaptation testing, and colour testing can help support the diagnosis. As discussed above, fluorescein angiography is particularly useful for evaluating sequelae, and both fluorescein angiography and electroretinography are useful for following the course of the disease.

\section{Differential diagnosis}

The differential diagnosis for birdshot retinochoroidopathy includes a variety of disorders, none of which has a significant association with HLA-A29.

Syphilis or tuberculosis may present with vitritis and hypopigmented fundus lesions. ${ }^{44}$ Constitutional signs and symptoms, and appropriate testing (RPR or VDRL and FTA-ABS or MHA-TP for the former; chest $x$ ray and PPD with anergy panel for the latter) can help to differentiate these uveitides from birdshot.

Sarcoidosis can mimic birdshot, ${ }^{46}$ particularly when systemic signs and symptoms are absent, which is not unusual for ocular sarcoidosis. ${ }^{47}$ Again, appropriate tests can provide diagnostic assistance: serum angiotensin converting enzyme, 24 hour urine calcium, anergy panel (anergy may occur in sarcoidosis), pulmonary function tests (diffusion capacity may be subnormal in sarcoidosis; not useful in smokers), chest $x$ ray, gallium scan of the head, neck, and mediastinum, biopsy of any conjunctival granuloma or of the palpebral lobe of the lacrimal gland if it is enlarged.

Lymphoma should be considered, ${ }^{47}$ especially in immunocompromised individuals. Like sarcoidosis, systemic 
findings may be absent. The diagnosis is aided by magnetic resonance imaging of the head and orbits with and without contrast, lumbar puncture for cerebrospinal fluid analysis, and diagnostic vitrectomy with cytological and immunohistological studies.

Some white dot syndromes can be confused with birdshot, particularly multiple evanescent white dot syndrome (MEWDS), multifocal choroiditis and panuveitis, and acute posterior multifocal placoid pigment epitheliopathy (AMPPE). However, these diseases usually occur in younger patients than those with birdshot, and the degree of vitritis, appearance and distribution of the fundus lesions, and course of the disease differ from birdshot. Unlike birdshot, MEWDS usually is unilateral, presents with minimal or no vitritis and posterior pole lesions that are smaller and whiter than those of birdshot, and spontaneously improves within 6 weeks. ${ }^{48}$ Multifocal choroiditis and panuveitis syndrome differs from birdshot in that inflammation is very prominent, the fundus lesions are smaller and better defined, hyperpigmentation around the optic disc and fundus lesions is common, and both ERG abnormalities and night blindness are uncommon. ${ }^{49}$ Elevated Epstein-Barr virus titres have been associated with this syndrome. ${ }^{50}$ As distinct from birdshot, AMPPE occurs acutely, and presents with minimal vitritis and placoid fundus lesions in the posterior pole that usually resolve within 6 weeks, leaving RPE changes and good visual acuity. Furthermore, fluorescein angiography is distinctive and shows early blockage and late hyperfluorescence associated with the lesions. ${ }^{51}$

Other diseases that are less likely to be confused with birdshot than those above, but which are in the differential diagnosis, are pars planitis, Harada's disease, sympathetic ophthalmia, presumed ocular histoplasmosis, and choroidal pneumocystosis.

\section{Course}

The course of birdshot retinochoroidopathy comprises exacerbations and remissions. ${ }^{11}$ Thus, it is difficult to evaluate the effect of treatment.

\section{Treatment}

There is no specific optimal treatment. Generally, systemic treatment is not warranted in binocular patients unless visual acuity diminishes below 20/40 bilaterally, which most commonly is due to cystoid macular oedema. One notable exception to this rule applies to subretinal neovascularisation threatening central vision. It always warrants consideration of prompt laser photocoagulation. ${ }^{47}$

\section{CORTICOSTEROIDS}

Periocular and systemic steroids are the mainstay of therapy. Periocular steroids are used for disease with asymmetric severity and for exacerbations in patients on systemic therapy. ${ }^{47}$

Initial improvement in vision may occur with periocular steroid injection or high dose prednisone $(1 \mathrm{mg} / \mathrm{kg} /$ day $)$. However, neither treatment is consistently effective..$^{1-4} 818$ One study indicates that less than $15 \%$ of patients who need treatment and respond to prednisone can remain exacerbation free on $\leqslant 20 \mathrm{mg} /$ day ${ }^{42}$ With tapering, exacerbations are common, ${ }^{42}$ but may be prevented by a very slow taper.

\section{CYCLOSPORINE}

Cyclosporine probably is effective because it compromises CD4 lymphocyte function, which is thought to mediate birdshot. It has proved to be an effective treatment alone $(2-5 \mathrm{mg} / \mathrm{kg} /$ day $)$ or with low dose prednisone $\quad<20$ $\mathrm{mg} /$ day) or azathioprine $(1.5-2 \mathrm{mg} / \mathrm{kg} /$ day $) .{ }^{16} 425253$ In one study it reduced vitreous inflammation and stabilised or improved vision in $83.3 \%$ (20) of eyes. ${ }^{53}$

\section{CYTOTOXIC AGENTS}

Cytotoxic agents have been used early in the disease because progressive impairment of retinal integrity (for example, retinal thinning and vascular attenuation) and function can occur despite suppression of inflammation, possibly due to triggering of apoptosis. However, these agents do not appear to alter the clinical course of birdshot and their potential side effects are hazardous for long term treatment, which some birdshot patients require. ${ }^{47}$

\section{OTHER TREATMENTS}

Aromatic retinoids reportedly resolved the ocular and dermatological problems of one patient with both birdshot and psoriasis. ${ }^{20}$

Non-steroidal anti-inflammatory agents or radiation have not been effective. ${ }^{485}$

\section{Prevention}

Birdshot retinochoroidopathy cannot be prevented. Preventive measures are directed at avoiding vision threatening complications. All patients complaining of floaters need close follow up, even if central visual acuity is stable. ${ }^{11}$

\section{Prognosis}

The prognosis is variable. About $20 \%$ of patients have self limited disease. These patients have spontaneous regression of intraocular inflammation and maintain 20/20 vision with persistence of fundus lesions. ${ }^{11}$ However, some believe that few patients maintain good visual acuity without treatment. ${ }^{11}$

Of 27 patients followed for 5-13 years, visual acuity worsened in $52 \%$, remained stable in $30 \%$, and improved in $18 \%$. Final acuity was $\geqslant 20 / 40$ in at least one eye in $67 \%$ and $\leqslant 20 / 200$ in the better sighted eye in $22 \%$. All patients were treated except for two whose vision remained stable. Cystoid macular oedema contributed towards visual loss in $41 \%$. Other causes of visual loss included macular epiretinal membrane, subretinal neovascularisation, macular scar, retinal detachment, vitreous opacities, vitreous haemorrhage, cataract, optic atrophy, and secondary glaucoma. ${ }^{3}$ Early in the disease, vitreous haemorrhage and floaters were major causes of visual loss. ${ }^{3}$

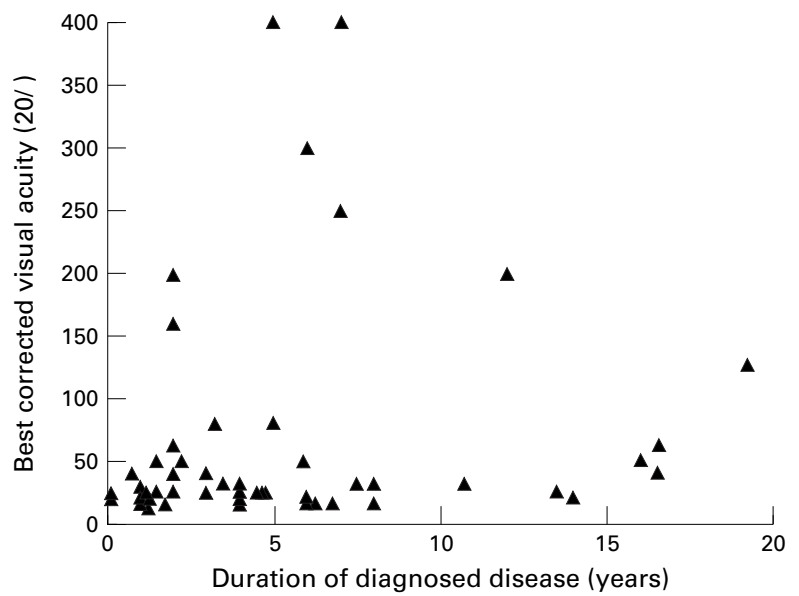

Figure 6 Best corrected visual acuity of the better sighted eye versus duration of diagnosed disease for the NEI birdshot population, except for one eye with finger counting vision and three patients (six eyes) whose duration of diagnosed disease was unavailable $(n=55)$. 


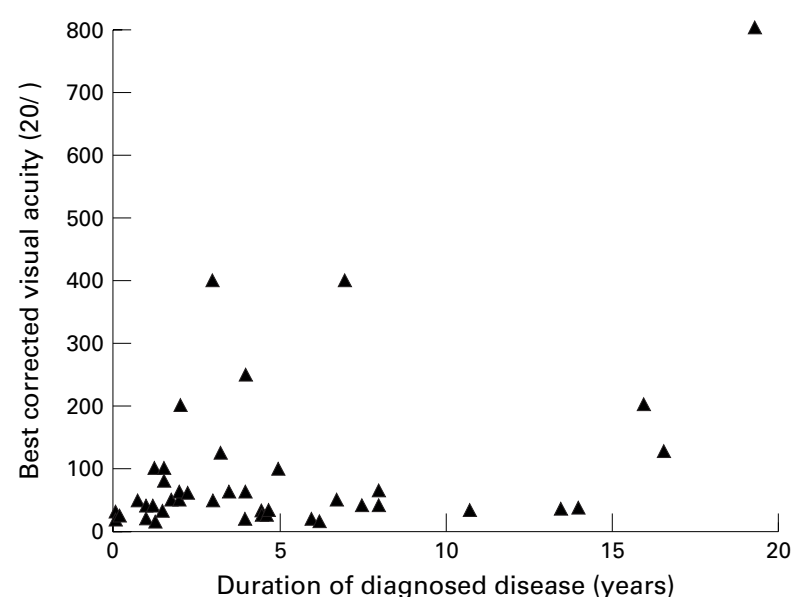

Figure 7 Best corrected visual acuity of the worse sighted eye versus duration of diagnosed disease for the NEI birdshot population, except for five eyes with finger counting vision, two eyes with hand movement vision, two eyes that had been enucleated due to sequelae of cataract extraction, and three patients (six eyes) whose duration of diagnosed disease was unavailable $(n=45)$.

Visual improvement in 13 eyes of 62 patients followed for 1 month to 13 years was attributed to clearing of vitreous haemorrhage (three eyes), cataract extraction (three eyes), laser photocoagulation of subretinal neovascularisation (two eyes), and regression of inflammation (five eyes). ${ }^{3}$

Of the NEI's 59 patients, visual acuity was $\geqslant 20 / 20$ in at least one eye of 18 patients $(31 \%)$. These patients had been diagnosed with birdshot for 1 month to 14 years. Seven of them had received no treatment. Visual acuity was $\geqslant 20 / 40$ in at least one eye in 41 patients $(70 \%)$, and it was $\leqslant 20 / 200$ in the better sighted eye in seven patients $(12 \%)$. The latter group had been diagnosed with birdshot for 5-12 years, and three had received no treatment. Figures 6 and 7 show best corrected visual acuity versus duration of diagnosed disease for better and worse sighted eyes respectively in the NEI population.

\section{Conclusion}

Findings in the NEI population confirm previous observations regarding most aspects of the disease, including the age, race, sex, and general health of patients; disease sequelae; and results of laser flare photometry, ICG angiography, electrophysiological testing, and colour vision testing. Visual field testing results also corroborated previous reports except that peripheral visual field constriction was not evident.

Symptom incidence has not been reported previously. Both this information and the implication from the NEI population that all birdshot patients, both active and inactive, exhibit the following features should help to make the diagnosis, which is determined clinically: (1) minimal, if any, anterior segment inflammation; (2) vitritis without snowbanking and/or vitreous debris; and (3) typical birdshot lesions (described above under "Typical presentation") bilaterally or typical birdshot lesions unilaterally in the setting of a middle aged whites of northern European extraction with HLA-A29 positivity and both blue-yellow dyschromatopsia and nyctalopia in the eye without lesions.

The unknown pathogenesis of birdshot contributes towards the difficulty in finding optimal treatment for the disease, and the relapsing course of the disease makes evaluation of treatment difficult. Nevertheless, findings in the NEI population suggest that $70 \%$ of patients have visual acuity $\geqslant 20 / 40$. About $12 \%$ develop vision $\leqslant 20 / 200$.

Financial support: none Proprietary interest: none
ALICE T GASCH JANINE A SMITH SCOTT M WHITCUP

National Eye Institute, National Institutes of Health, Bethesda, MD, USA

Correspondence to: Scott $M$ Whitcup, MD, National Eye Institute, National Institutes of Health, 10 Center Drive, Bldg 10 Rm 10N202, Bethesda, MD 20892-1858, USA.

1 Gass JD. Vitiliginous chorioretinitis. Arch Ophthalmol 1981;99:1778-87.

2 Kaplan HJ, Aaberg TM. Birdshot retinochoroidopathy. Am f Ophthalmol 1980;90:773-82.

3 Priem HA, Oosterhuis JA. Birdshot chorioretinopathy: clinical characteristics and evolution. Br F Ophthalmol 1988;72:646-59.

4 Ryan SJ, Maumenee AE. Birdshot retinochoroidopathy. Am f Ophthalmol 1980;89:31-45.

5 Baarsma GS, Kijlstra A, Oosterhuis JA, et al. Association of birdshot retinochoroidopathy and HLA-A29 antigen. Doc Ophthalmol 1986;61:267-9.

6 Baarsma GS, Priem HA, Kijlstra A. Association of birdshot retinochoroidopathy and HLA-A29 antigen. Curr Eye Res 1990;9:63-8.

7 LeHoang P, Ozdemir N, Benhamou A, et al. HLA-A29.2 subtype associated with birdshot retinochoroidopathy. Am f Ophthalmol 1992;113:33-5.

8 Nussenblatt RB, Mittal KK, Ryan S, et al. Birdshot retinochoroidopathy associated with HLA-A29 antigen and immune responsiveness to retinal S-antigen. Am f Ophthalmol 1982; 94:147-58.

9 Priem HA, Kijlstra A, Noens L, et al. HLA typing in birdshot chorioretinopathy. Am f Ophthalmol 1988;105:182-5.

10 Franceschetti A, Babel J. La chorio-rétinite en "täches de bougie," manifestation de la maladie de Besnier-Boeck. Ophthalmologica 1949;118:701-10.

11 LeHoang P, Ryan SJ. Birdshot retinochoroidopathy. In: Pepose JS, Holland GN, Wilhelmus KR, eds. Ocular infection and immunity. St Louis: Mosby, 1995:570-8

12 Amalric $\mathrm{P}$, Cuq G. Une forme très particulière de choriorétinopathie en grains de riz. Bull Soc Ophtalmol Fr 1981;81:131-4.

13 Henderly DE, Genstler AJ, Smith RE, et al. Changing patterns of uveitis. Am f Ophthalmol 1987;103:131-6.

14 Brucker AJ, Deglin EA, Bene C, et al. Subretinal choroidal neovascularization in birdshot retinochoroidopathy. Am f Ophthalmol 1985;99:40-4.

15 de Waal LP, Lardy NM, van der Horst AR, et al. HLA-A29 subtypes and birdshot chorioretinopathy. Immunogenetics 1992;5:51-3.

16 LeHoang P, Girard B, Deray G, et al. Cyclosporine in the treatment of birdshot retinochoroidopathy. Transplant Proc 1988;20(suppl 4):128-30.

17 Laroche L, Saraux H, Quentel G, et al. Bird-shot chorioretinopathy après décollement de rétine: origine auto-immune probable. Bull Soc Ophtalmol Fr 1983;83:1245-7.

18 Fuerst DJ, Tessler HH, Fishman GA, et al. Birdshot retinochoroidopathy. Arch Ophthalmol 1984;102:214-19.

19 Yoshioka T, Yoshioka H, Tanaka F. Birdshot retinochoroidopathy as a new ocular sign of sarcoidosis. Nippon Ganka Gakkai Zasshi 1983;87:283-8.

20 Hesse S, Berbis P, Chemila JF, et al. Psoriasis and birdshot chorioretinopathy: response to aromatic retinoids. Dermatology 1993;187:137-9.

21 Heaton JM, Mills RP. Sensorineural hearing loss associated with birdshot retinochoroidopathy. Arch Otolaryngol Head Neck Surg 1993;119:680-1.

22 Dannenberg AL, Garrison RJ, Kannel WB. Incidence of hypertension in the Framingham study. Am f Public Health 1988;78:676-9.

23 Soubrane G, Bokobza R, Coscas G. Late developing lesions in birdshot retinochoroidopathy. Am f Ophthalmol 1990;109:204-10.

24 Ryan SJ, Dugel PU, Stout JT. Birdshot retinochoroidopathy. In: Ryan SJ, ed. Retina. 2nd ed. St Louis: Mosby, 1994:1677-85.

25 Borondes MJ, Fastenberg DM, Schwartz, et al. Peripheral retinal neovascularization in birdshot retinochoroidopathy. Ann Ophthalmol 1989;21:306-8.

26 Henkind P. Ocular neovascularization: the Krill Memorial Lecture. Am $\mathfrak{f}$ Ophthamol 1978;85:287-301.

27 Caballero-Presencia A, Diaz-Guia E, Lopez-Lopez JM. Acute anterior ischemic optic neuropathy in birdshot retinochoroidopathy. Ophthalmologica 1988;196:87-91.

28 Guex-Crosier Y, Pittet N, Herbort CP. Evaluation of laser flare-cell photometry in the appraisal and management of intraocular inflammation in uveitis. Ophthalmology 1994;101:728-35.

29 Chang B, Lumbroso L, Rabb MF, et al. Birdshot chorioretinopathy. In: Yannuzzi LA, Flower RW, Slakter JS, eds. Indocyanine green angiography. St Louis: Mosby, 1997:231-8.

30 Hirose T, Katsumi O, Pruett RC, et al. Retinal function in birdshot retinochoroidopathy. Acta Ophthalmol 1991;69:327-37.

31 Priem HA, De-Rouck A, De-Laey JJ, et al. Electrophysiologic studies in birdshot chorioretinopathy. Am f Ophthalmol 1988;106:430-6.

32 Yang SY. Population analysis of class I HLA antigens by one-dimensional isoelectric focusing gel electrophoresis: workshop summary report. In: isoelectric focusing gel electrophoresis: workshop summary report. In:
Dupont $\mathrm{B}$, ed. Immunobiology of $H L A$. New York: Springer-Verlag, 1989:309-31.

33 Tabary T, Prochnicka-Chalufour A, Cornillet P, et al. HLA-A29 subtypes and "birdshot" choroido-retinopathy susceptibility: a possible "resistance motif" in the HLA-A29.1 molecule. C R Acad Sci III 1991;313:599-605.

34 Fich M, Rosenberg T. Birdshot retinochoroidopathy in monozygotic twins. Acta Ophthalmol 1992;70:693-7.

35 de-Smet MD, Yamamoto JH, Mochizuki M, et al. Cellular immune responses of patients with uveitis to retinal antigens and their fragments. Am $\mathcal{F}$ Ophthalmol 1990;110:135-42.

36 Jobin D, Thillaye B, de-Kozak Y, et al. Severe retinochoroidopathy: variations of humoral and cellular immunity to $S$-antigen in a longitudinal study. Curr Eye Res 1990;9(Suppl): 91-6.

37 Faure JP. Autoimmunity and the retina. Curr Top Eye Res 1980;2:215-302.

38 Faure JP, LeHoang P, Takano S, et al. Uvéo-rétinite expérimentale induite par l'antigène $S$ rétinien chez le singe. $\mathcal{F} \mathrm{Fr}$ Ophtalmol 1981;4:465-72.

39 Nordmann JP, De-Kozak Y, LeHoang P, et al. Cyclosporine therapy of guinea-pig autoimmune uveoretinitis induced with autologous retina. $\mathcal{F}$

40 Nussenblatt RB, Kuwabara T, de Monasterio F, et al. S-antigen uveitis in primates: a new model for human, disease. Arch Ophthalmol 1981;99:1090- 
41 Kalsow CM, Wacker WB. Pineal reactivity of anti-retina sera. Invest Ophthalmol Vis Sci 1977;16:181-4.

42 Opremcak EM. Birdshot retinochoroiditis. In: Albert DM, Jakobiec FA, eds, Principles and practice of ophthalmology. Philadelphia: WB Saunders, 1994:475-80

43 Feltkamp TEW. Ophthalmological significance of HLA associated uveitis. Eye 1990;4:839-44

44 Schlaegel TF, Kao SF. A review of 28 presumptive cases of syphilitic uveitis. Am fO Ophthalmol 1982;93:412-4.

45 Abrams J, Schlaegel TF. The role of isoniazid therapeutic test in tuberculous uveitis. Am f Ophthalmol 1982;94:511-5.

46 Brod RD. Presumed sarcoid choroidopathy mimicking birdshot retinochoroidopathy. Am f Ophthalmol 1990;109:357-8.

47 Nussenblatt RB, Whitcup SM, Palestine AG. Uveitis: fundamentals and clinical practice. 2nd ed. St Louis: Mosby, 1996:290, 325-33.

48 Jampol LM, Sieving PA, Pugh D, et al. Multiple evanescent white dot syndrome: clinical findings. Arch Ophthalmol 1984;102:671-4.
49 Nozik RA, Dorsch W. A new chorioretinopathy associated with anterior uveitis. Am f Ophthalmol 1973;76:758-62.

50 Tiedeman JS. Epstein-Barr viral antibodies in multifocal choroiditis and panuveitis. Am f Ophthalmol 1987;103:659-63.

51 Gass JDM. Acute posterior multifocal placoid pigment epitheliopathy. Arch Ophthalmol 1968;80:177-85.

52 Nussenblatt RB, Palestine AG, Chan CC. Cyclosporine A: therapy in the treatment of' intraocular inflammatory disease resistant to systemic corticosteroids and cytotoxic agents. Am f Ophthalmol 1983;96:275-82.

53 Vitale AT, Rodriguez A, Foster CS. Low-dose cyclosporine therapy in the treatment of birdshot retinochoroidopathy. Ophthalmology 1994;101:82231

54 Hofmann HM, Feicht W. Birdshot chorioretinopathy: systemic therapy with corticosteroids and nonsteroidal anti-inflammatory drugs. Klin Monatsbl Augenheilkd 1990;197:159-61. 